\title{
Intestinal parassitosis in Reggio Emilia during 2007: critical analysis of results
}

\author{
Carlotta Guidetti, Carlo Capatti, Lidia Ricci, Luigi Vecchia \\ U.O. di Microbiologia, Arcispedale Santa Maria, Nuova Reggio Emilia, Italy
}

Key Words: parassitosi intestinali, protozoi, elminti, immigrati

Parassitosi intestinali nell'area reggiana nel corso del 2007: analisi critica dei risultati

\section{SUMMARY}

In 2007 were analyzed in 2032 samples from 1970 subjects: coprparassitologiacal standard examination was performed in 1916 of the subjects and scotch-test in the remaining 54. Of the 1970 subjects examined, 81 , equivalent to $4.1 \%$, resulted positive for one or more parasites (both pathogens and / or of questionable pathogenicity). Pathogenic parasites were found in 38 subjects, equivalent to $46.9 \%$ of the total positive: in 20 cases (24.7\%) cysts of Giardia intestinalis were found in 7 cases (8.6\%) it was Enterobius vermicularis, in another $5(6.2 \%)$ there was Hymenolepis nana, in 2 (2.4\%) Trichuris trichiura, in I (I.2\%) Ancylostoma / necator, in I (I.2\%), Dicrocoelium dendriticum, in I (I.2\%) Dipylidium caninum, and in I (I.2\%) Ascaris lumbricoides. Protozoa of doubtful pathogenicity, either alone or in combination with pathogens, were found in 54 subjects: Blastocystis hominis in 30 cases (36.9\%), Entamoeba coli in 15 (18.8\%), Endolimax nana in 10 (12.3\%), Entamoeba histlytica dispar in 4 (4.9\%), lodamoeba butschlii in I (I.2\%), Entamoeba hartmanni in I (I.2\%). These data represent only a first frame of the possible scope of the phenomenon, both in terms of diagnostic possibilities and in terms of real etiological significance in order to qualify the diagnosis, and to ensure careful monitoring of the phenomenon. We also wanted to evaluate retrospectively the existing diagnostic potential before embarking on some organizational and procedural changes in the field of diagnostic parasitology.

Received June 3, 2009

Accepted June 6, 2009

\section{INTRODUZIONE}

Attualmente le parassitosi intestinali autoctone nel nostro paese sono piuttosto rare $(2,16,17,30)$. Le prevalenze sono molto basse soprattutto per gli elminti, tra i quali i soli nematodi Enterobius vermicularis e Strongyloides stercoralis sembrano ancora rivestire un ruolo di una certa importanza, il primo in particolare nei bambini in età scolare $\mathrm{e}$ il secondo nei soggetti anziani $(9,31)$. Per ciò che riguarda i protozoi, Giardia intestinalis $e$ Dientamoeba fragilis rappresentano i principali protozoi patogeni intestinali presenti in Italia sebbene la loro prevalenza venga spesso sottostimata, sia per l'aspecificità dei sintomi, sia per un errato approccio operativo diagnostico $(20,25,28)$. Tra i protozoi viene spesso segnalato il saprofita opportunista Blastocystis hominis, il cui ruolo patogeno è tuttora controverso $(5,7,21,29)$. La segnalazione, infine, di protozoi non patogeni (amebe e flagellati) è solitamente occasionale, soprattutto in virtù degli elevati standard igienici ormai raggiunti più o meno ovunque e delle positive abitudini idro-alimentari attualmente esistenti nel Paese $(1,6,16,27)$.

L'interesse e l'attenzione della e nella diagnostica parassitologica devono tuttavia essere sempre vivi in relazione a vari fattori tra cui la crescente presenza di immigrati nel nostro paese (soprattutto da Paesi africani ed asiatici, dall' Europa dell'Est, dal centro e sud- America), il consistente aumento dei viaggi (per turismo, lavoro, volontariato) e le adozioni internazionali, che hanno fatto riemergere questo tipo di patologie sia sul piano strettamente clinico che nel campo della sanità pubblica in generale (12, 23, 24, 26, 32).

Alla luce di quanto detto, risulta utile la ricerca di parassiti nelle feci delle seguenti categorie di individui: soggettti portatori di disturbi intestinali aspecifici (sovente da lungo tempo); pazienti con diarrea verosimilmente o presumibilmente infetti-

\section{Corresponding author: Carlotta Guidetti}

Laboratorio di Microbiologia, Arcispedale Santa Maria, Nuova Reggio Emilia

Via Risorgimento, 80; 42100 - Reggio Emilia, Italy - Tel: 3498466603

E.mail: carlottaguidetti@libero.it 
Tabella I. Totale dei soggetti parassitati, distribuzione e quantificazione

\begin{tabular}{|c|c|c|c|c|c|c|}
\hline & \multicolumn{2}{|c|}{ Autoctoni } & \multicolumn{2}{|c|}{ Immigrati } & \multicolumn{2}{|c|}{ Totale } \\
\hline Soggetti parassitati & 26 & $32,1 \%$ & 55 & $67,9 \%$ & 81 & $4,1 \% *$ \\
\hline \multicolumn{7}{|l|}{ Soggetti parassitati da } \\
\hline I patogeno & 2 & $2,5 \%$ & 24 & $29,7 \%$ & 26 & $32,2 \%$ \\
\hline \multicolumn{7}{|l|}{ Soggetti parassitati da } \\
\hline 2 patogeni & I & $1,2 \%$ & 0 & - & 1 & $1,2 \%$ \\
\hline \multicolumn{7}{|l|}{ Soggetti parassitati da } \\
\hline I patogeno e I o più non patogeni & 6 & $7.4 \%$ & 5 & $6,1 \%$ & II & $13,5 \%$ \\
\hline \multicolumn{7}{|l|}{ Soggetti parassitati da } \\
\hline I o più non patogeni & 17 & $21 \%$ & 26 & $32,1 \%$ & 43 & $53,1 \%$ \\
\hline
\end{tabular}

*percentuale dei soggetti parassitati (8I) sul totale dei soggetti esaminati (1970)

Tabella 2. Classificazione e distribuzione dei parassiti patogeni e di dubbia patogenicità negli 8 I soggetti parassitati

\begin{tabular}{|c|c|c|c|c|c|c|}
\hline PARASSITI PATOGENI & \multicolumn{2}{|c|}{ Autoctoni } & \multicolumn{2}{|c|}{ Immigrati } & \multicolumn{2}{|c|}{ Totale $^{\wedge}$} \\
\hline ELMINTI & 2 & $2,5 \%$ & 16 & $19,7 \%$ & 18 & $22,2 \%$ \\
\hline Enterobius vermicularis & 2 & $2,5 \%$ & 5 & $6, I$ & 7 & $8,6 \%$ \\
\hline Ascaris lumbricoides & 0 & - & 1 & $1,2 \%$ & $\mathrm{I}$ & $1,2 \%$ \\
\hline Trichuris trichiura & 0 & - & 2 & $2,5 \%$ & 2 & $2,6 \%$ \\
\hline Ancylostoma/Necator & 0 & - & $\mathrm{I}$ & $1,2 \%$ & $\mathrm{I}$ & $1,2 \%$ \\
\hline Hymenolepis nana & 0 & - & 5 & $6,2 \%$ & 5 & $6,2 \%$ \\
\hline Dicrocoelium dendriticum & 0 & - & $\mathrm{I}$ & $1,2 \%$ & $\mathrm{I}$ & $1,2 \%$ \\
\hline Dipylidium caninum & 0 & - & 1 & $1,2 \%$ & 1 & $\mathrm{I}, 2 \%$ \\
\hline PROTOZOI & 7 & $8,6 \%$ & 13 & $16,1 \%$ & 20 & $24,7 \%$ \\
\hline Giardia intestinalis & 7 & $8,6 \%$ & 13 & $16,1 \%$ & 20 & $24,7 \%$ \\
\hline $\begin{array}{l}\text { PARASSITI NON PATOGENI } \\
\text { (o DI DUBBIA PATOGENICITÀ) }\end{array}$ & \multicolumn{2}{|c|}{ Autoctoni } & \multicolumn{2}{|c|}{ Immigrati } & \multicolumn{2}{|c|}{ Totale ${ }^{\wedge}$} \\
\hline Blastocystis hominis & 19 & $23,4 \%$ & 11 & $13,5 \%$ & 30 & $36,9 \%$ \\
\hline Entamoeba coli & 0 & - & 15 & $18,8 \%$ & 15 & $18,8 \%$ \\
\hline Endolimax nana & 3 & $3,7 \%$ & 7 & $8,6 \%$ & 10 & $12,3 \%$ \\
\hline lodamoeba buetschlii & $\mathrm{I}$ & $1,2 \%$ & 0 & - & $\mathrm{I}$ & $\mathrm{I}, \mathbf{2} \%$ \\
\hline Entamoeba hartmanni & $\mathrm{I}$ & $1,2 \%$ & 0 & - & $\mathrm{I}$ & $1,2 \%$ \\
\hline Entamoeba histolyticaldispar ${ }^{\circ}$ & 3 & $3,7 \%$ & 1 & $1,2 \%$ & 4 & $4,9 \%$ \\
\hline
\end{tabular}

$\wedge$ La percentuale è stata calcolata sul totale $(8 \mathrm{I})$ dei soggetti parassitati

${ }^{\circ}$ Entamoeba histolytica/dispar è stata interpretata come commensale, potenzialmente patogeno

\section{DISCUSSIONE}

Dai risultati ottenuti dallo studio retrospettivo riportato, emerge che la prevalenza di parassitosi intestinali nell'area di Reggio Emilia, sembra essere relativamente bassa (4.1\%). Soltanto il 2 \% circa, infatti, dei 1970 soggetti che si sono sottoposti ad un esame copro parassitologico standard o scotch test sono risultati parassitati da protozoi o elminti la cui patogenicità è indiscussa, mentre il rimanente $2 \%$ era parassitato da protozoi considerati "commensali" o il cui ruolo patogeno è tuttora in discussione. Questo potrebbe però in parte essere correlato ai limitati percorsi e tecniche diagnostiche applicate: il mancato utilizzo di colorazioni permanenti e appositi terreni di coltura ha sicuramente causato la sottostima di parassiti il cui ruolo patogeno non è in discussione, quali Dientamoeba fragilis e Strongyloides stercoralis, per nulla rari nel nostro paese $(10,13)$. Dei due gruppi di soggetti analizzati, gli autoctoni presentano frequenze più basse di parassitosi rispetto agli immigrati stranieri nei quali sono stati rilevati parassiti insoliti nell'area reggiana: Ancylostoma/Necator, Ascaris lumbricoides, Dipylidium caninum e Dicrocoelium dendriticum, (sia pure, in questi ultimi due casi interpretati come uova di transito passivo) (18). Nel complesso negli 81 soggetti parassitati, la presenza di protozoi $(77.8 \%)$, risultava di gran lunga maggiore rispetto agli elminti (22.2 \%). Tra i protozoi patogeni, l'unico identificato è stato Giardia intestinalis, mentre tra i protozoi non patogeni Blastocystis hominis (presente nel $36.9 \%$ dei soggetti positivi) prevale, immediatamente seguito da Entamoeba coli (presente nel $18.8 \%$ ). E. histolytica/dispar è stata diagnosticata all'esame microscopico e la sua presenza confermata col metodo immunocromatografico (Triage ${ }^{\circledR}$ parasite panel Biosite HQ and Manufacturing Campus, Mera Misa, California, USA). In assenza di sintomatologia specifica e di trofozoiti senza fenomeni di fagocitosi a carico di emazie E. histolyrica/dispar è stata 
classificata tra i protozoi non patogeni. La differenziazione tra E. histolytica e E. dispar, morfologicamente identiche, si basa su criteri particolari che non possono essere alla portata della maggior parte dei laboratori, nostro incluso. Tra gli elminti, solo Enterobius vermicularis e Hymenolepis nana prevalgono sulle altre specie, riscontrate solo in 1 o 2 soggetti. Relativamente alla clinica, purtroppo, a causa dell'attuale sistema organizzativo-gestionale, con centri prelievi anche periferici cui afferiscono i campioni biologici per le indagini parassitologiche, attualmente non corredati da schede clinico-anamnestiche adeguate, non è stato possibile correlare retrospettivamente il rilevamento dei parassiti alla sintomatologia clinica dei soggetti parassitati. Tali dati vogliono pertanto rappresentare soltanto un primo fotogramma di quale possa essere la portata del fenomeno, sia in termini di possibilità diagnostiche sia in termini di significatività eziologica reale. L'applicazione di colorazioni permanenti, ricerche mirate per Strongyloides stercoralis, l'utilizzazione di congrui formati delle richieste e quindi più attente informazioni clinico-anamnestico-epidemiologiche, l'introduzione di algoritmi diagnostici basati sul quesito medico di partenza, l'aspetto del campione ed i risultati del primo esame microscopico costituiscono un insieme di elementi che successivamente alla presente indagine sono stati adottati nella nostra realtà operativa onde potere qualificare la diagnostica, e garantire un' attenta sorveglianza del fenomeno.

\section{BIBLIOGRAFIA}

1. Antico A, Pellizzer G, Romeo I, Corà R. Due casi di amebiasi nell'Ovest Vicentino: valutazioni epidemiologiche e cliniche; Microbiol. Med. 1998; 13: 351-353

2. Barbaro G. B, Cavallino A. M, Gianotti A, Patrone C, Robello A. Esami coproparassitologici: analisi di una casistica quinquennale raccolta nel territorio del Ponente Genovese; GIMMOC 2001; vol. V, $\mathrm{n}^{\circ}$ 3: $18-20$

3. Bernieri F, Crotti D, Galli D, Raglio A. Manuale illustrato di diagnostica parassitologica. Pavia: Edizioni Selecta Medica 2001

4. Bernieri F, Casella P, Crotti D. et al. Linee Guida Operative per la diagnosi delle Parassitosi Intestinali. Microbiol. Med. 2005; 20: 39-46

5. Bernieri F, Galli D, Crotti D, Del Sante M. Studio policentrico dell'Associazione Microbiologi Clinici Italiani-Comitato di Studio di Parassitologia (AMCLICoSP) sulla diffusione delle parassitosi,intestinali in Italia: parassitosi intestinali nell’immigrato. Giorn. It. Med. Trop. 1996; 1: 97-99

6. Bossi L, Perego L. Infezione da Cryptosporidium spp. in soggetti HIV positivi: una casistica. Microbiol. Med. 1991; 6: 81-84
7. Camporese A. Blastocystis hominis: una parassitosi sottovalutata. Primi dati di un'indagine. Microbiol. Med. 1992; 7: 26-31

8. Cancrini G. Parassitologia medica illustrata. Roma: Lombardo Editore 1996

9. Crotti D, D’Annibale M.L, Medori M.C, Fonzo G, Ubaldi M. Parassitosi intestinali autoctone nell'area del Perugino: considerazioni di metodologia diagnostica e prevalenze nel corso del 2001. GIMMOC. 2003; vol. VII, $n^{\circ}$ 1: 12-20

10. Crotti D, D’Annibale ML, Rossi S, Strongyloides stercoralis e strongiloidiasi in Umbria: analisi di una casistica decennale. Microbiologia Medica 2005; vol 20, $n^{\circ} 4: 250-256$

11. Crotti D, D’Annibale ML, Medori MC. Diagnostica microbiologica ed epidemiologica locale delle enteriti acute e protratte: risultati relativi all'anno 2002. Giorn It Mal Inf 2003; 9;205-212

12. Crotti D, D’Annibale ML. Dientamoeba fragilis e dientamoebiasi: aspetti di parassitologia clinica e diagnostica di laboratorio. Parassitologia 2001; 3:135-138

13. Crotti D, D’Annibale ML. Parassitosi intestinali autoctone: ruolo di Dientamoeba fragilis nelle infezioni umane. Ann Ig 2007; 19: 27-34

14. Crotti D, Raglio A, Galli D, Bernieri F. L'esame coproparassitologico nei bambini. Indicazioni e indagini raccomandate. Quaderni acp 2004; 12: 39-42

15. Crotti D. Enterobiasis in Italy today: a Primary School's experience. Microbiol. Med. 2008; 23: 64-67

16. Crotti D. Parassitosi intestinali autoctone nella II metà degli anni '90: considerazioni critiche diagnostiche. Microbiol. Med. 2001; 17: 7-13

17. Crotti D, Del Sante M. Parassitosi intestinali autoctone nella popolazione del Perugino: prevalenze e considerazioni diagnostico-epidemiologiche. Giorn. It. Mal. Inf. 1197; 3: 83-88

18. D’Annibale ML. Infestazione spuria da Dicrocoelium dendriticum. Microbiol. Med. 2004; 19: 399-400

19. De Carneri I. Parassitologia generale e umana. XI Ed. Milano: Casa editrice Ambrosiana 1992

20. Fazii P, Scassa E, Cosentino L. Epidemiologia della Giardiasi nel pescarese. Parassitologia, 1998; 40 (Suppl. 1): 54-57

21. Francavilla R, Iaiani G, Franchi C, Galanti G. Amebiasi intestinale: la nostra esperienza su una patologia riemergente. Giorn. It. Mal. Inf. 1198; 4:363-369.

22. Garcia LS. Practical Guide to Diagnosric Parasitology. Washington D.C.: ASM Press 1999; 23.

23. Johnson EH, WindsorJJ, Graham Clark C. Emerging from obscurity: biological, clinical, and diagnostic aspects of Dientamoeba fragilis. Clin Microbiol rev 2004; 17: 553-70

24. Lainson R, da Silva BAM. Intestinal Parasites of some diarrhoeic HIV-positive individuals in North Brazil, with particular reference to Isospora belli Wenyon, 1923 and Dientamoeba fragilis Jepps \&Dobell 1918. Mem Inst Oswaldo Cruz 1999; 94 (5):611-3

25. Libman MD, Gyorkos TW, Kokoskin E, MacLean JD. Detection of Pathogenic Protozoa in the Diagnostic Laboratory: Result Reproducibility, Specimen Pooling, and Competency Assessment. JCM 2008; 46: 2200-05

26. Norberg A, Nord CE, Evengaard B. Dientamoeba fragilis -a protozoal infection which may cause severe bowel di stress. CMI 2003; 9:65-8

27. Pampiglione S, Canestri Trotti G. Guida allo studio della Parassitologia. Società Editrice Esculapio, Bologna, 1990 
va (più spesso “diarrea del viaggiatore”); soggetti con ipereosinofilia e/o con anemia; soggetti asintomatici ma con almeno uno dei seguenti fattori di rischio: provenienza o rientro da paesi endemici per parassitosi; individui a contatto stretto con soggetti dimostratisi parassitati (scuole, famiglie, collettività chiuse); anziani da sottoporre a terapie cortisoniche o immunosoppressive (in tali circostanze però la ricerca è mirata per il solo $S$. stercoralis); pazienti immunocompromessi $(3,8,19$, 22).

Scopo del presente studio è quello di valutare la prevalenza di parassitosi intestinale nell'area Reggiana, e la sua distribuzione in base alla nazionalità dei pazienti, pur con i limiti intrinseci legati alle metodologie utilizzate al riguardo, e fornire informazioni comunque utili per capirne le dimensioni onde implementarne, se possibile, la qualità diagnostica.

\section{MATERIALI E METODI}

L'indagine a valenza retrospettiva si riferisce all'anno 2007 ed è stata condotta su campioni fecali pervenuti al laboratorio di Microbiologia della Azienda Ospedaliera di Reggio Emilia, appartenenti a soggetti residenti o domiciliati nei comuni di Reggio Emilia e provincia. Sono stati analizzati 2032 campioni provenienti da 1970 soggetti (autoctoni e immigrati stranieri, sia ambulatoriali che ricoverati), con sospetto clinico di parassitosi intestinali. I campioni fecali sono stati raccolti secondo le istruzioni operative del laboratorio che prevedono: sospensione di antibiotici e chemioterapici 72 ore prima del prelievo, raccolta di quantità adeguate di campione (poco più di una noce, quando solide), e la consegna preferibilmente entro 2 ore dall'emissione, o conservate al massimo 72 ore in frigorifero. In caso di impossibilità di consegna tempestiva del campione i campioni fecali sono stati posti in flaconi contenenti sostanze conservanti (formalina al 10\%) (3). Ai soggetti con diarree acute o protratte, è stato esaminato usualmente un solo campione fecale, rappresentato da feci liquide, poltacee o comunque non formate $(9,11,16)$.

L'esame copro parassitologico standard (ECPS) eseguito su campioni fecali di 1916 soggetti comprendeva: osservazioni macroscopiche a fresco del campione fecale, per la ricerca di eventuali proglottidi di tenie o vermi adulti; esame microscopico diretto e dopo colorazioni estemporanee iodate (lugol), utile per evidenziare sia gli elminti, ma soprattutto i trofozoiti dei protozoi; esame microscopico dopo arricchimento specifico, ovvero la concentrazione formolo - etilacetato (FEA), finalizzato alla ricerca di uova di elminti e alle cisti di Giardia intestinalis e dei principali protozoi non patogeni, seguendo le raccomandazioni indicate in letteratura $(4,13,14)$.

La ricerca mirata di Enterobius vermicularis, in 54 soggetti, per lo più bambini, è stata eseguita con la tecnica dello scotch test, presso l'ambulatorio dell'ospedale (15).

\section{RISULTATI}

Dei 1970 soggetti esaminati, 81, pari al $4.1 \%$, sono risultati positivi per 1 o più parassiti (patogeni e/o non patogeni). Di questi, 55, pari al 67,9 \%, erano immigrati stranieri, e 26, pari al 32,1\% erano italiani. I soggetti che presentavano parassiti di indubbia patogenicità erano 38 (29 immigrati e 9 italiani), pari al 46,9 \% del totale dei positivi. Nel gruppo dei soggetti parassitati da patogeni, 26 erano positivi per un solo parassita; 1 è risultato positivo per due parassiti patogeni e 11 sono risultati postivi per più parassiti (comprendenti un patogeno in associazione con 1 o più parassiti non patogeni o di dubbia patogenicità). I soggetti infestati da soli parassiti non patogeni erano 43, pari al $53.1 \%$ del totale dei positivi; tra questi, 40 sono risultati positivi per un solo parassita non patogeno, 3 sono risultati postivi a 2 o più non patogeni in associazione (Tabella 1). Complessivamente negli 81 soggetti positivi sono stati riscontrati 99 parassiti.

Tra le specie parassitarie individuate sono stati reperiti: elminti in 18 casi (pari al $22.2 \%$ dei positivi), protozoi patogeni in 20 soggetti (pari al 24.7 $\%$ dei positivi), protozoi non patogeni o di dubbia patogenicità (da soli o in associazione tra di loro 0 con patogeni) in 54 soggetti (66.6 \% dei positivi) per un totale di 61 parassiti.

Sono stati identificati i seguenti elminti: Enterobius vermicularis in 7 soggetti, Hymenolepis nana in 5, Trichuris trichiuria in 2, Ancylostoma/Necator in 1, Ascaris lumbricoides in 1, Dicrocoelium dendriticum (le cui uova sono state ritenute di transito) in 1, Dipylidium caninum in 1 (anch'esso espressione verosimile di ingestione passiva di uova).

Tutti i protozoi patogeni identificati appartenevano alla specie Giardia intestinalis in forma cistica. Sono poi stati riscontrati i seguenti protozoi non patogeni (o di dubbia patogenicità, quanto per il primo citato): 30 Blastocystis hominis (36.9 \% dei soggetti positivi), 15 Entamoeba coli (18.8 \% dei soggetti positivi), 10 Endolimax nana (12.3\% dei soggetti positivi) 1 Iodamoeba butschlii (1.2 \%),1 Entamoeba hartmanni (1.2 \%), Entamoeba histolytica/dispar, classificata tra i protozoi commensali, solo potenzialmente patogeni in assenza di sintomatologia clinica specifica, è stata riscontrata in 4 soggetti (4.9\%) (Tabella 2$)$. 
28. Pica R. Indagine di laboratorio sulle parassitosi: esperienza dell'Ospedale san Giovanni di Roma. Parassitologia 1998; 40: 134

29. Rossi M. R, Cirelli C, Pantaleoni M. Incidenza di protozoi intestinali in pazienti HIV positivi con diarrea acuta e cronica. Microbiol. Med. 1992; 7: 123

30. Ricci L, Barbieri Palmieri F. Aspetti epidemiologici delle parassitosi intestinali in una USL dell'Emilia
Romagna. Microbiol. Med. 1993; 8: 35-38

31. Ricci L, Barbieri Palmieri F. Strongiloidiasi disseminata: descrizione di due casi. Giorn. It. Mal. Inf. 1997; 3: $112-114$

32. Stark D, Beebe N, Marriot D, Ellis J, Harkness J. Detection of Dientamoeba fragilis in fresh stool specimens using PCR. Int J Parasitol 2005; 35:57-62 